\title{
Marcadores sorológicos do vírus da hepatite B em mulheres jovens atendidas pelo Programa de Saúde da Família em Vitória, Estado do Espírito Santo, 2006
}

\author{
Serological markers for hepatitis B virus in young women attended by \\ the Family Health Program in Vitória, Espírito Santo, 2006 \\ Nínive Camillo de Figueiredo', Kimberly Page-Shafer ${ }^{2}$, \\ Fausto Edmundo Lima Pereira ${ }^{1}$ e Angélica Espinosa Miranda ${ }^{1}$
}

\begin{abstract}
RESUMO
O objetivo deste estudo foi determinar a frequiência dos marcadores sorológicos para hepatite B e de fatores de risco associados com a infecção pelo vírus B em mulheres jovens, residentes em Vitória, ES, onde a vacinação em recém-nascidos e em adolescentes foi iniciada em 1994 e 2000 , respectivamente. Estudo populacional, por amostragem, realizado em três regiões de saúde de Vitória em 2006. Foi realizada entrevista e pesquisa de HBsAg, anti-HBc e anti-HBs. De 1.200 mulheres selecionadas, 1.029 (85,7\%) participaram do estudo. A mediana de idade foi 23 anos (distância interquartil 20-26 anos) e 93,2\% tinham mais de quatro anos de escolaridade. Quarenta e três (4,2\%) mulheres (IC95\% 2,97\%-5,43\%)] apresentaram anti-HBc total positivo e 9 (0,9\%) (IC95\% 0,4\%-1,6\%)] HBsAg. Houve 466 (45,3\%) testes (IC95\% 42,2\%-48,4\%)] anti-HBs positivos dos quais 427 eram anti-HBc e HBsAg negativas. A única variável independentemente associada com anti-HBc (+) foi renda mensal de até 4 salários mínimos [OR =2,6 (IC95\% 1,06-6,29) ]. Os dados mostram baixa prevalência do vírus B e de seus fatores de risco mais conhecidos. A prevalência do antiHBs com anti-HBC e HBsAg negativos reflete a cobertura vacinal do Município neste grupo (43,7\%). Não foi possível determinar fatores de risco significativos para a aquisição do vírus hepatite B nessa população.
\end{abstract}

Palavras-chaves: Hepatite B. Mulheres jovens. Prevalência. Fatores de risco.

\section{ABSTRACT}

The aim of this study was to determine the frequencies of serological markers for hepatitis B and risk factors associated with HBV infection among young women living in Vitória, Espírito Santo, where vaccination for newborns and adolescents started in 1994 and 2000, respectively. This was a population-based study performed by sampling in three health regions of Vitória in 2006. Interviews were held and HBsAg, anti-HBc and anti-HBs were investigated. Out of 1,200 women selected, 1,029 (85.7\%) were enrolled. The median age was 23 years (interquartile range: 20-26 years) and 93.2\% had had more than four years of schooling. Forty-three women $(4.2 \%$; 95\%CI 2.97\%-5.43\%) were positive for anti-HBc total, and nine, for HBsAg (0.9\%; 95\%CI 0.4\%-1.6\%)]. There were 466 positive anti-HBs tests ( $45.3 \% ; 95 \% \mathrm{CI} 42.2 \%-48.4 \%)$, of which 427 were negative for anti-HBc and HBsAg. Monthly income of up to four minimum monthly salaries was the only variable independently associated with positive anti-HBc tests $(\mathrm{OR}=$ 2.6: 95\%CI 1.06-6.29). These data show low prevalence of the hepatitis B virus and its better-known risk factors. The prevalence of positive anti-HBs tests with negative anti-HBc and HBsAg tests reflects the vaccine coverage in the municipality in this group (43.7\%). It was not possible to determine any significant risk factors for hepatitis B virus acquisition among this population.

Key-words: Hepatitis B. Young women. Prevalence. Risk factors.

\footnotetext{
1. Programa de Pós-Graduação em Doenças Infecciosas, Núcleo de Doenças Infecciosas, Universidade Federal do Espírito Santo., Vitória, ES. 2.Center for AIDS Prevention Studies, Department of Medicine, University of California, San Francisco, USA.

Apoio financeiro: AIDS International Training in Research Program (Fogarty International Center) D43W00003 e ICOHRTA Brazil Scientist Program (Fogarty International Center) D43TW005799

Endereço para correspondência: Dra. Nínive Camillo de Figueiredo. Núcleo de Doenças Infecciosas/UFES. Av. Marechal Campos 1468, Maruípe, $29040-091$ Vitória, ES.

Tel: 5527 3335-7504

e-mail: ninive-figueiredo@ig.com.br

Recebido para publicação em 13/05/2008

Aceito em 12/11/2008
} 
A hepatite B é uma enfermidade de distribuição universal que afeta ambos os sexos, mas que adquire especial importância entre mulheres pela alta transmissão materno-infantil ${ }^{13}{ }^{17}$. A infecção também pode ser adquirida por transmissão horizontal através de contato sexual, compartilhamento de seringas, exposição ocupacional, transfusão de sangue contaminado e convívio familiar ${ }^{511}$. 0 padrão de transmissão do vírus da hepatite B (VHB) está relacionado com a taxa de prevalência. Em áreas de baixa endemicidade a transmissão sexual e percutâneas são preponderantes, nas de alta endemicidade a maioria das infecções ocorrem por transmissão vertical ${ }^{10}$. A despeito do conhecimento das principais vias de transmissão, pelo menos $20 \%$ dos casos de infecção aguda permanecem com fonte obscura de contato ${ }^{21}$.

A prevalência da infecção tem grandes variações geográficas, com regiões de alta prevalência (mais de $8 \%$ de portadores do HBsAg), média prevalência (entre $2 \%$ e $7 \%$ ) e baixa prevalência $(\text { menos de } 2 \%)^{1}$. No Brasil, a prevalência é heterogênea, com a região Amazônica, o Espírito Santo e o oeste de Santa Catarina consideradas zonas de alta e média prevalência ${ }^{14}$ e as demais regiões com índices baixos, semelhantes à América do Norte e Europa Ocidental 247151920 .

No Espírito Santo a prevalência do vírus também é heterogênea, com municípios de prevalência média, especialmente os de colonização italiana, sendo os demais de prevalência baixa ${ }^{18}$. Em Vitória, onde existem os maiores Hospitais Gerais públicos do estado, é freqüente o diagnóstico de doenças crônicas ligadas ao VHB, especialmente hepatite crônica e carcinoma hepatocelular ${ }^{8}$, e a prevalência do anti-HBc está em torno de $12 \%$ no maior banco de sangue público do estado9.

Em razão de ter áreas de média prevalência do VHB, o Espírito Santo foi incluído no programa de vacinação para hepatite B em 1994, e hoje a cobertura vacinal completa é de 77,5\% no estado e de $83,9 \%$ na capital (SEMUS: dados não publicados, 2005); entretanto, quando estratificada por faixa etária, a cobertura é bastante heterogênea. Entre os adolescentes de 11 a 14 anos, $37,6 \%$ haviam recebido as três doses da vacina e apenas $16 \%$ dos adultos entre 20 e 59 anos completaram a vacinação (SEMUS: dados não publicados, 2005).

Conhecer a prevalência da infecção e a situação vacinal em mulheres jovens é importante para se ter idéia do potencial da transmissão vertical a fim de elaborar medidas de intervenção. Por essas razões os objetivos do presente estudo foram: a) determinar a prevalência de marcadores do vírus da hepatite $B$ em mulheres jovens residentes em Vitória; b) determinar o índice de proteção vacinal através da pesquisa do anti-HBS e c) descrever os fatores de risco em relação à hepatite $B$.

\section{MATERIAL E MÉTODOS}

Foi realizado um estudo de base populacional, por amostragem, em três regiões de saúde de Vitória, onde o Programa de Saúde da Família (PSF) já estava implantado e que dispunham de uma lista completa dos domicílios. As regiões incluídas foram:
Maruípe, São Pedro e Continental com habitantes pertencentes às classes média alta, média e média baixa.

Constituição da amostra. 0 cadastro do PSF continha registro de 10.660 mulheres de 18 a 29 anos, que habitavam as regiões descritas, anteriormente. Utilizando uma tabela de números aleatórios foi obtida uma amostra de 1.200 mulheres. 0 tamanho da amostra foi calculado para estimar a taxa de infecção persistente pela hepatite $\mathrm{B}$, com um intervalo de $95 \%$ de confiança, na população de estudo. Tomou-se como base para cálculo uma prevalência de HBsAg de 1\% em gestantes residentes em Vitória ${ }^{12}$, o que gerou um número de 990 mulheres. Admitindo-se uma perda de $20 \%$, foram selecionadas 1.200 mulheres. A amostra teve 30,7\% de mulheres da Região Continental, 51\% de Maruípe e 18,3\% de São Pedro, já que essas proporções representavam as proporções de mulheres, na faixa etária do estudo, em cada região, em relação ao total das residentes nas três regiões do Município. A coleta de dados, realizada por enfermeiras do PSF, foi feita de março a dezembro de 2006.

Questionário. 0 questionário utilizado foi uma forma modificada daquele utilizado na pesquisa The Young Women's Survey ${ }^{16}$. 0 questionário foi validado e foram mantidas as perguntas que informavam sobre a) dados sócio-demográficos (idade, escolaridade, ocupação, estado civil e renda); b) dados clínicos (antecedentes de vacinação, história de DST, contracepção, gravidez e abortos); c) informações sobre práticas sexuais e sobre comportamentos de risco (uso de preservativos, número de parceiros sexuais, prostituição, abuso sexual, uso de álcool e drogas, transfusão de sangue). Todas as perguntas contidas no questionário eram fechadas.

Pesquisa dos marcadores da infecção com o vírus da hepatite B. Foram pesquisados o antígeno de superfície do vírus da hepatite B (HBsAg), os anticorpos anti-antígeno do core (anti-HBc) e anti-antígeno de superfície (anti-HBs), usando testes comerciais (Auszyme Monoclonal EIA, Corzyme EIA, Ausab EIA, Laboratórios Abbott, Rio de Janeiro, Brasil). Os testes foram realizados pelo Laboratório Central da Secretaria Municipal de Saúde de Vitória, seguindo as instruções do fabricante.

Análise de dados. A análise estatística foi realizada utilizando-se o SPSS versão 11.5 para Windows. Inicialmente, foi realizada uma análise descritiva, incluindo distribuição de frequiência para variáveis qualitativas e cálculo de média e desvio - padrão para variáveis quantitativas. A prevalência de infecção pelo vírus B foi estimada pela frequiência do diagnóstico em questão, sendo calculado o correspondente intervalo de confiança de $95 \%$. As possíveis associações entre infecção pelo vírus da hepatite B e comportamentos de risco ou variáveis demográficas e clínicas foram testadas através de testes de qui-quadrado. odds ratio e intervalos de confiança foram calculados para estimar o grau de associação entre a infecção e os potenciais fatores de risco. Análise multivariada de regressão logística foi utilizada para estimar o efeito de uma variável, a mesmo tempo em que se controla o efeito das demais.

Aspectos éticos. Este projeto foi submetido e aprovado pelo Comitê de Ética em Pesquisa do Centro de Ciências da Saúde da Universidade Federal do Espírito Santo e pelo Comitê de Ética 
em Pesquisa da Universidade da Califórnia, San Francisco. Todas as mulheres que aceitaram participar do projeto foram informadas dos objetivos da pesquisa, assinaram um termo de consentimento livre e esclarecido e receberam acompanhamento médico quando diagnosticadas com a infecção.

\section{RESULTADOS}

Entre as 1.200 mulheres selecionadas, 1.029 participaram do estudo, o que representa $85,7 \%$ da amostra inicialmente selecionada, com mediana de idade de 23 anos (distância interquartil (DIQ) de 20-26 anos).

Quarenta e três (4,2\%) mulheres (IC95\% 2,97\%-5,43\%) foram consideradas expostas ao vírus da hepatite B (VHB) por apresentarem anti-HBc total positivo e $9(0,9 \%)$ eram portadoras do HBsAg. (IC95\% 0,4\%-1,6\%)] 0 anti-HBs foi positivo (título superior a 10UI, conforme especificação do método utilizado) em 466 (45,3\%) mulheres (IC95\% 42,2\%-48,4\%).

A Tabela 1 descreve os dados demográficos e marcadores de hepatite B em mulheres jovens atendidas pelo PSF de Vitória, de acordo com a região de residência. Chama a atenção a alta $(93,2 \%)$ frequiência de escolaridade acima de quatro anos de estudo. Grande parte da população das regiões de São Pedro e Maruípe ganhava até quatro salários mínimos, enquanto $72,1 \%$ das mulheres da região continental relataram renda maior que quatro salários mínimos. Na Tabela 2 estão os dados relacionados com os fatores de risco para a infecção. 0 fator mais freqüente foi o uso de drogas ilícitas não injetáveis $(17,8 \%)$ e 0 menos freqüente o uso de drogas injetáveis $(0,5 \%) ; 87,9 \%$ das participantes relataram ser sexualmente ativas.

$\mathrm{Na}$ análise bivariada, a variável que se associou com a infecção pelo VHB (anti-HBc total positivo) foi a renda mensal de até quatro salários mínimos $[0 \mathrm{R}=2,49(\mathrm{IC} 95 \% 1,10-5,66)]$. Não houve associação de anti-HBc total com estado marital, número de parceiros sexuais, ou uso de preservativo na última relação sexual. 0 relato de prática de prostituição, antecedentes de DST e transfusão sangüínea também não mostraram significância estatística. Nenhuma das três mulheres, que relataram fazer uso de drogas injetáveis, estava infectada pelo vírus B (Tabela 3).

No modelo final de regressão logística, a variável renda mensal até quatro salários mínimos permaneceu associada com a presença de anti-HBc total $[\mathrm{OR}=2,6$ (IC95\% 1,06-6,29) ].

Tabela 1 - Dados demográficos e marcadores de hepatite B em mulheres jovens atendidas pelo Programa de Saúde da Família de Vitória, de acordo com o total e a região de residência.

\begin{tabular}{|c|c|c|c|c|c|c|c|c|}
\hline \multirow[b]{2}{*}{ Variáveis } & \multicolumn{2}{|c|}{ Total } & \multicolumn{2}{|c|}{ São Pedro } & \multicolumn{2}{|c|}{ Maruípe } & \multicolumn{2}{|c|}{ Continental } \\
\hline & $\mathrm{n}^{\mathrm{o}}$ & $\%$ & $\mathrm{n}^{\mathrm{o}}$ & $\%$ & $\mathrm{n}^{\mathrm{o}}$ & $\%$ & $\mathrm{n}^{\underline{0}}$ & $\%$ \\
\hline \multicolumn{9}{|l|}{ Idade (em anos) } \\
\hline 18 a 24 & 657 & 63,8 & 120 & 65,9 & 335 & 59,8 & 202 & 70,4 \\
\hline 25 a 29 & 372 & 36,2 & 62 & 34,1 & 225 & 40,2 & 85 & 29,6 \\
\hline \multicolumn{9}{|c|}{ Escolaridade (em anos) } \\
\hline$\leq 4$ & 70 & 6,8 & 15 & 8,2 & 50 & 8,9 & 5 & 1,7 \\
\hline$>4$ & 959 & 93,2 & 167 & 91,8 & 510 & 91,1 & 282 & 98,3 \\
\hline \multicolumn{9}{|l|}{ Estado marital } \\
\hline casada/amasiada & 604 & 58,7 & 103 & 56,6 & 266 & 47,5 & 235 & 81,9 \\
\hline não casada & 425 & 41,3 & 79 & 43,4 & 294 & 52,5 & 52 & 18,1 \\
\hline \multicolumn{9}{|c|}{ Renda mensal (salário mínimo) } \\
\hline$\leq 4$ & 700 & 68,0 & 140 & 76,9 & 480 & 85,7 & 80 & 27,9 \\
\hline$>4$ & 329 & 32,0 & 42 & 23,1 & 80 & 14,4 & 207 & 72,1 \\
\hline \multicolumn{9}{|c|}{ Anticorpos anti-antígeno do core } \\
\hline positivo & 43 & 4,2 & 7 & 3,8 & 21 & 3,8 & 15 & 5,2 \\
\hline negativo & 986 & 95,8 & 175 & 96,2 & 539 & 96,3 & 272 & 94,8 \\
\hline \multicolumn{9}{|c|}{ Antígeno de superfície do vírus da hepatite B } \\
\hline negativo & 1020 & 99,1 & 180 & 98,9 & 558 & 99,6 & 282 & 98,3 \\
\hline \multicolumn{9}{|c|}{ Anti-antígeno de superfície } \\
\hline positivo & 466 & 45,3 & 55 & 30,2 & 282 & 50,4 & 129 & 44,9 \\
\hline negativo & 563 & 54,7 & 127 & 69,8 & 278 & 49,6 & 158 & 55,1 \\
\hline \multicolumn{9}{|l|}{ Vacinação hepatite B* } \\
\hline $\operatorname{sim}$ & 356 & 34,6 & 46 & 25,3 & 202 & 36,1 & 108 & 37,6 \\
\hline não & 276 & 26,8 & 83 & 45,6 & 86 & 15,4 & 107 & 37,3 \\
\hline não sei & 397 & 38,6 & 53 & 29,1 & 272 & 48,6 & 72 & 25,1 \\
\hline
\end{tabular}

* A informação não pôde ser confirmada pelo cartão de vacinação, pois muitas das participantes não tinham mais o cartão. 
Tabela 2 - Dados comportamentais de risco para hepatite B em mulheres jovens atendidas pelo Programa de Saúde da Familia de Vitória, de acordo com o total e a região de residência.

\begin{tabular}{|c|c|c|c|c|c|c|c|c|}
\hline \multirow[b]{2}{*}{ Variáveis } & \multicolumn{2}{|c|}{ Total } & \multicolumn{2}{|c|}{ São Pedro } & \multicolumn{2}{|c|}{ Maruípe } & \multicolumn{2}{|c|}{ Continental } \\
\hline & $\mathrm{n}^{0}$ & $\%$ & $\mathrm{n}^{0}$ & $\%$ & $\mathrm{n}^{0}$ & $\%$ & $\mathrm{n}^{0}$ & $\%$ \\
\hline \multicolumn{9}{|l|}{ Uso drogas ilícitas } \\
\hline $\operatorname{sim}$ & 183 & 17,8 & 37 & 20,3 & 105 & 18,8 & 41 & 14,3 \\
\hline não & 846 & 82,2 & 145 & 79,7 & 455 & 81,3 & 246 & 85,7 \\
\hline \multicolumn{9}{|c|}{ Uso drogas injetáveis } \\
\hline $\operatorname{sim}$ & 5 & 0,5 & 3 & 1,6 & 1 & 0,2 & 1 & 0,3 \\
\hline não & 1.024 & 99,5 & 179 & 98,4 & 559 & 99,8 & 286 & 99,7 \\
\hline \multicolumn{9}{|c|}{ Transfusão sanguínea } \\
\hline $\operatorname{sim}$ & 30 & 2,9 & 7 & 3,8 & 19 & 3,4 & 4 & 1,4 \\
\hline não & 999 & 97,1 & 175 & 96,2 & 541 & 96,6 & 283 & 98,6 \\
\hline \multicolumn{9}{|l|}{ Abuso sexual } \\
\hline $\operatorname{sim}$ & 83 & 8,1 & 15 & 8,2 & 58 & 10,4 & 10 & 3,5 \\
\hline não & 946 & 91,9 & 167 & 91,8 & 502 & 89,6 & 277 & 96,5 \\
\hline \multicolumn{9}{|l|}{ Atividade sexual } \\
\hline $\operatorname{sim}$ & 904 & 87,9 & 170 & 93,4 & 513 & 91,6 & 221 & 77,0 \\
\hline não & 125 & 12,1 & 12 & 6,6 & 47 & 8,4 & 66 & 23,0 \\
\hline \multicolumn{9}{|l|}{ Parceiros vida* } \\
\hline 1 parceiro & 295 & 32,6 & 47 & 27,6 & 172 & 33,5 & 76 & 34,4 \\
\hline$>1$ parceiro & 609 & 67,4 & 123 & 72,4 & 341 & 66,5 & 145 & 23,8 \\
\hline \multicolumn{9}{|l|}{ Uso preservativo* } \\
\hline $\operatorname{sim}$ & 330 & 36,5 & 65 & 38,2 & 165 & 32,2 & 100 & 45,2 \\
\hline não & 574 & 63,5 & 105 & 61,8 & 348 & 67.8 & 121 & 54,8 \\
\hline \multicolumn{9}{|c|}{ História doenças sexualmente transmissíveis* } \\
\hline $\operatorname{sim}$ & 109 & 12,1 & 24 & 14,1 & 47 & 9,2 & 38 & 17,2 \\
\hline não & 795 & 87,9 & 146 & 85,9 & 466 & 90,8 & 183 & 82,8 \\
\hline \multicolumn{9}{|l|}{ Prostituição* } \\
\hline $\operatorname{sim}$ & 14 & 1,4 & 10 & 4,7 & 3 & 0,6 & 1 & 0,5 \\
\hline não & 890 & 98,6 & 160 & 95,3 & 510 & 99,4 & 220 & 99,5 \\
\hline
\end{tabular}

Tabela 3 - Associação de dados sociodemográficos e comportamentais com anticorpos anti-antígeno (anti-HBc) do core total em mulberes jovens de Vitória, ES.

\begin{tabular}{|c|c|c|c|c|c|c|c|c|c|}
\hline \multirow[b]{2}{*}{ Variáveis } & \multicolumn{2}{|c|}{ anti-HBc } & \multirow[b]{2}{*}{ OR $(\text { IC95\%) })^{1}$} & \multirow[b]{2}{*}{ Valor P } & \multirow[b]{2}{*}{ Variáveis } & \multicolumn{2}{|c|}{ anti-HBc } & \multirow[b]{2}{*}{ OR $(\text { IC95\% })^{1}$} & \multirow[b]{2}{*}{ Valor P } \\
\hline & positivo & negativo & & & & positivo & $\overline{\text { negativo }}$ & & \\
\hline Idade (em anos) & & & & & Prostituição $0^{2}$ & & & & \\
\hline 18 a 24 & 23 & 634 & $0,64(035-1,18)$ & 0,194 & $\operatorname{sim}$ & 0 & 12 & 3 & 1,000 \\
\hline 25 a 29 & 20 & 352 & 1,0 & & não & 39 & 853 & & \\
\hline Escolaridade (em anos) & & & & & História DST ${ }^{2}$ & & & & \\
\hline$\leq 0 \mathrm{a} 4$ & 3 & 67 & $1,03(0,31-3,41)$ & 1,000 & $\operatorname{sim}$ & 6 & 103 & $1,35(0,55-3,29)$ & 0,456 \\
\hline$>4$ & 40 & 919 & 1,0 & & não & 33 & 762 & 1 & \\
\hline Estado marital & & & & & Abuso sexual & & & & \\
\hline casada & 7 & 205 & $0,74(0,33-1,69)$ & 0,567 & $\operatorname{sim}$ & 3 & 80 & $1,15(0,35-3,80)$ & 0,554 \\
\hline não casada & 36 & 781 & 1 & & não & 40 & 906 & 1 & \\
\hline Renda mensal & & & & & Uso drogas não injetáveis & & & & \\
\hline$\leq 4 \mathrm{SM}$ & 36 & 644 & $2,49(1,10-5,66)$ & 0,029 & $\operatorname{sim}$ & 12 & 171 & $1,85(0,93-3,66)$ & 0,100 \\
\hline$>4 \mathrm{SM}$ & 7 & 322 & 1 & & não & 31 & 815 & 1 & \\
\hline Região de saúde & & & & & Drogas injetáveis & & & & \\
\hline Continental & 8 & 279 & 1 & 0,370 & Sim & 0 & 5 & 3 & 1,000 \\
\hline Maruípe & 27 & 533 & $1,77(0,79-3,94)$ & & Não & 43 & 981 & & \\
\hline São Pedro & 8 & 120 & $1,60(0,59-4,35)$ & & Transfusão sanguínea & & & & \\
\hline Parceiros sexuais ${ }^{2}$ & & & & & $\operatorname{sim}$ & 2 & 28 & $1,67(0,38-7,25)$ & 0,360 \\
\hline parceiro único & 16 & 279 & 1 & 0,295 & não & 41 & 958 & 1 & \\
\hline$>1$ parceiro & 23 & 586 & $1,46(0,76-2,81)$ & & & & & & \\
\hline \multicolumn{10}{|l|}{ Uso preservativo ${ }^{2}$} \\
\hline $\operatorname{sim}$ & 14 & 316 & $0,97(0,50-1,90)$ & 0,936 & & & & & \\
\hline não & 25 & 549 & 1 & & & & & & \\
\hline
\end{tabular}

'OR (IC95\%): odds ratio. ${ }^{2}$ dados relativos às 904 mulheres sexualmente ativas. ${ }^{3}$ cálculo realizado somente para Tabela $2 X 2$.

Anti-HBc: anticorpos anti-antígeno, OR (IC95\%): odds ratio (intervalo de confiança 95\%), DST: doença sexualmente transmissível, SM: salário mínimo. 


\section{DISCUSSÃo}

Trata-se do primeiro estudo com base populacional delineado para estudar a prevalência de marcadores de VHB em mulheres jovens (18 a 29 anos) no Brasil, em uma região onde a vacinação para esse vírus foi iniciada em recém-nascidos em 1994 e em adolescentes a partir de 2000 (SESA: dados não publicados, 2005). Na amostra estudada, 427 mulheres eram anti-HBs positivas (título acima de 10UI) e eram antiHBc negativas, perfil sorológico típico de pessoas vacinadas. Considerando que a pesquisa do anti-HBc foi positiva em 43 mulheres e o HBsAg em 9 mulheres que tiveram contacto com o vírus e provavelmente não eram vacinadas, pode-se admitir que a proteção vacinal na amostra estudada é de 43,7\% (427 anti-HBs positivas entre 977 negativas para os outros marcadores do VHB). Os dados permitem concluir que a vacinação de adolescentes tem uma razoável proporção de cobertura em Vitória, já que 43,7\% das mulheres até 29 anos têm níveis protetores de anti-HBs. Ainda que seja uma cobertura vacinal baixa, é maior do os $16 \%$ de cobertura estimada pela Secretaria de Saúde do Município de Vitória.

A prevalência do anti-HBc, que marca o contacto prévio com 0 vírus foi de $4,2 \%$ em toda a amostra. No entanto se retirarmos da amostra 0 grupo de mulheres que foi vacinado a prevalência vai para 7,2\% (43/596 mulheres), menor do que a observada nos doadores voluntários do banco de sangue em Vitória, que era de $12,8 \%$ em $2000^{\circ}$. Entretanto foi aproximada aos dados observados em outros estudos realizados na região sudeste ${ }^{4719}$.

0 HBsAg foi positivo em 9 (0,9\%) mulheres da amostra examinada. Se for considerado que grande parte da amostra foi vacinada na infância ou adolescência, a prevalência da infecção persistente no soro nas mulheres não vacinadas foi de $1,3 \%$, frequiência aproximada ao observado em outros estudos realizados em gestantes s $^{3671213} \mathrm{e}$ do observado em estudo populacional realizado em São Paulo 7 .

A análise dos fatores de risco para infecção pelo VHB neste estudo confirma a baixa prevalência dos marcadores estudados, já que foram pouco freqüentes os relatos de transfusão de sangue, uso de drogas injetáveis e prostituição. Os fatores de risco mais frequientemente relatados foram a história pregressa de DST e 0 relato de mais de um parceiro sexual. Eles também foram mais freqüentes no grupo de mulheres com marcadores positivos para $o$ vírus B (HBsAg e ou anti-HBc). A multiplicidade de parceiros, o não uso de preservativos e a prática comercial da atividade sexual são conhecidos fatores de associação com as doenças sexualmente transmissíveis ${ }^{1}$. Na avaliação dos fatores conhecidos como de risco para infecção pelo VHB, apenas a renda familiar abaixo de 4 salários mínimos se correlacionou positivamente com a presença do anti-HBc. Estudo realizado por Silveira e cols ${ }^{19}$ também mostrou uma clara associação entre o baixo padrão sócio-econômico e a soropositividade para o VHB no Brasil. A ausência de correlação com fatores como o maior número de parceiros sexuais, uso de drogas injetáveis e prostituição pode estar relacionado à baixa frequiência desses fatores na amostra estudada.
Embora 0 estudo transversal não seja 0 ideal na determinação das possíveis vias de contágio do VHB, sua aplicação se justifica porque conhecer a soroprevalência em mulheres jovens, na faixa de maior fecundidade, é importante para demonstrar a susceptibilidade da população à forma mais grave de transmissão. Devido à baixa prevalência do VHB e dos fatores de risco nesta amostra, e à cobertura vacinal, 0 número de mulheres infectadas não foi suficiente para que se encontrasse associação estatística entre as variáveis independentes estudadas e a infecção pelo VHB. A possibilidade de ter ocorrido viés de resposta não pode ser descartada devido à tendência geral de se dar respostas socialmente aceitáveis.

0 conhecimento das formas de contágio facilita o estudo das variáveis envolvidas no risco de transmissão, entretanto tornase um desafio estudar populações onde estes mecanismos são desconhecidos e a prevalência é baixa ${ }^{15}$. Os dados do presente estudo oferecem um panorama da prevalência de marcadores sorológicos do vírus B entre as mulheres jovens de Vitória. Eles mostram uma baixa prevalência do vírus B e de seus fatores de risco mais conhecidos, a prevalência encontrada de anti-HBs reflete uma boa cobertura vacinal no Município. Entretanto, a multiplicidade das formas de exposição, o período de incubação prolongado e a forma assintomática na maioria dos portadores permanecem como desafios no estudo da infecção pelo vírus B.

\section{REFERÊNCIAS}

1. Alter MJ. Epidemilogy of hepatitis B in Europe and worldwide. Journal of Hepatology 39:64-69, 2003.

2. Brasil LM, Fonseca JC, Souza RB, Braga WSM, Toledo LM. Prevalência de marcadores para o vírus da hepatite B em contatos domiciliares no Estado do Amazonas. Revista da Sociedade Brasileira de Medicina Tropical 36:565-570, 2003.

3. Cardoso DDP, Faria EL, Azevedo MSP, Queiroz DAO, Martins RMB, Souza TT, Ferreira M. Soroepidemiologia para o vírus da hepatite B em gestantes/ parturientes e sua transmissão para recém-nascidos em Goiânia-GO. Revista da Sociedade Brasileira de Medicina Tropical 29:349-353, 1996.

4. Clemens SAC, Fonseca JT, Azevedo T, Cavalcanti A, Silveira TR, Castilho MC, Clemens R. Soroprevalência para hepatite A e B em quatro centros do Brasil. Revista da Sociedade Brasileira de Medicina Tropical 33:1-10, 2000.

5. Davis LG, Weber DJ, Lemon SM. Horizontal transmission of hepatitis B virus. The Lancet 33:889-893, 1989.

6. Duarte G, Pinhata MMM, Lemos C, Quintana SM, Paschioni MC, Motta MSF. Hepatite B e gravidez. Revista Brasileira de Ginecologia e Obstetrícia 19:9-12, 1997.

7. Figueiró Filho EA, Senefonte FR, Lopes AH, Morais 00, Souza Júnior VG, Maia TL, Duarte G. Frequiência das infecções pelo HIV-1, rubéola, sífilis, toxoplasmose, citomegalovirus, herpes simples, hepatite $\mathrm{B}$, hepatite $\mathrm{C}$, doença de Chagas e HTLV I/II em gestantes, do Estado do Mato Grosso do Sul. Revista da Sociedade Brasileira de Medicina Tropical 40:181-187, 2007.

7. Focaccia R, Conceição OJ , Sette Jr H, Sabino E, Bassit L, Nitrini DR, Lomar AV, Lorenço R, Souza FV , Kiffer CR, Santos EB, Gonzales MP, Sáez-Alquézar A, Riscal JR, Fischer D. Estimated prevalence of viral hepatitis in the general population of the municipality of São Paulo, measured by a serologic survey of stratified, randomized and residence-based population. Brazilian Journal of Infectious Diseases 2: 269-284, 1998.

8. Gonçalves CS, Pereira FEL, Gomes MPZ. Aspectos epidemiológicos de 190 casos de carcinoma hepatocelular (CHC) diagnosticados em Vitória, ES. Gastroenterologia e Endoscopia Digestiva 16:165-170, 1997. 
9. Gonçalves PL, Gonçalves CS, Folleto S, Pereira FEL. Marcadores de infecção do vírus $B$ em doadores de sangue com sorologia positiva para o vírus $C$ da Hepatite. Gastrenterologia e Endoscopia Digestiva 20 (supl): S70, 2001.

10. Mahorrey FJ. Update on diagnosis, management and prevention of hepatitis virus infeccion. Clinical Microbiologycal Reviews 12:351-366, 1999.

11. Margolis HS, Alter MJ, Hadler SC. Hepatitis B: evolving epidemiology and implications for control. Seminars in Liver Diseases 11:84-92, 1991.

12. Miranda AE, Alves MC, Neto RL, Areal KR, Gerbase AC. Seroprevalence of HIV, $\mathrm{HBV}$ and syphilis in women at their first visit to public antenatal clinics in vitória, Brazil. Sexually Transmitted Diseases 28:710-713, 2001.

13. Oliveira ND, Kopelman BI, Mundin HC, Campos TMA. Prevalência de gestantes portadoras do vírus B e transmissão perinatal. Journal of Pediatrics 69:53-60, 1993.

14. Ministério da Saúde, Fundação Nacional de saúde, Programa Nacional de Imunização: PNI 25 anos: Brasília, MS, 1998.

15. Rosini N, Mousse D, Spada C, Treitinger A. Seroprevalence of HBsAg, anti-HBc and anti-HCV in southen Brazil, 1999-2001. Brazilian Journal of Infectious Diseases 7:262-267, 2003.

16. Ruiz JD, Molitor F, McFarland W, Klausner J, Lemp G, Page-Shafer K, Parikh-Patel A, Morrow S, Sun RK. Prevalence of HIV infection, sexually transmited diseases, and hepatitis and related risk behavior in young women living in low-income neighborhoods of northern California. The Western Journal of Medicine 172:368-373, 2000.

17. Schweitzer IL. Vertical transmisson of the hepatitis B surface antigen. American Journal of Medicine Science 270:287-291, 1975.

18. Pereira AM, Andrade RLM. Inquérito epidemiológico de soroprevalência do vírus B da hepatite no estado do Espírito Santo, 1994-96. Secretaria de Estado da Saúde do Espírito Santo - SPEI/SESA. Boletim Epidemiológico SESA, 2001; http://www.saude.es.gov.br. (acessado em 31/Jul/2007), 2001.

19. Silveira TR, Fonseca JC, Rivera L, Fay OH, Tapia R, Santos JI, Urdeneta E, Clemens SA. Hepatitis B seroprevalence in Latin America. Revista Panamericana de Salud Publica 6:378-383, 1999.

20. Souto FJD. Distribuição da hepatite B no Brasil: atualização do mapa epidemiológico e proposições para seu controle. Gastroenterologia e Endoscopia Digestiva 18:143-150, 1999.

21. Van der Eijk AA, Niesters HG, Götz HM, Janssen HL, Schalm SW, Osterhaus AD, Man RA. Paired quantitative measurements of hepatitis B virus DNA in saliva, urine and serum of chronic hepatitis B pacients. Journal Clinical Virology 29:92-94, 2004. 